\title{
Animal Study
}

\section{e The Incorporation of Platelet-rich Plasma into Calcium Phosphate Cement Enhances Bone Regeneration in Osteoporosis}

\author{
Ah-Reum Cho, MD, PhD ${ }^{1,2}$, Hae-Kyu Kim, MD, PhD ${ }^{1,2}$, Jae-Young Kwon, MD, PhD1, \\ Tae-Kyun Kim, MD, PhD ${ }^{1}$, Yun-Mi Choi, MD', and Kyung-Hoon Kim, MD, PhD ${ }^{1}$
}

From: ${ }^{1}$ Department of Anesthesia and Pain Medicine, School of Medicine, Pusan National University; ${ }^{2}$ Medical Research Institute, Pusan National University Hospital, Busan, Republic of Korea

Address Correspondence: Kyung-Hoon Kim, MD, PhD Pain Clinic, Pusan National University Yangsan Hospital, Mulgeum-eup, Yangsan 626-770 Republic of Korea

E-mail: pain@pusan.ac.kr

Disclaimer: There was no external funding in the preparation of this manuscript. Conflict of interest: Each author certifies that he or she, or a member of his or her immediate

family, has no commercial association (i.e., consultancies, stock ownership, equity interest, patent/licensing arrangements, etc.) that might pose a conflict of interest in connection with the submitted manuscript.

Manuscript received: 03-19-2014 Accepted for publication: 07-29-14

Free full manuscript: www.painphysicianjournal.com
Background: Polymethyl methacrylate (PMMA) bone cement is widely used for osteoplasty. However, previous studies have demonstrated the adverse effects of PMMA due to its excessive stiffness and heat production. Recently, calcium phosphate cement (CPC) that overcomes those negative effects has been successfully applied in osteoplasty. The potential problem of CPC is markedly less initial stiffness. It leads to progressive, repeated collapse in the treated vertebra before CPC has been replaced by new bone that would provide substantial improvement in compressive strength and stiffness. The activated platelets in platelet-rich plasma (PRP) release a high concentration of growth factors which play an important role in bone healing.

Objective: To investigate whether PRP could accelerate the osteoconduction of CPC and enhance the bone strength of the treated vertebra in an animal model.

Study Design: Controlled animal study.

Setting: Laboratory animal study,

Methods: Thirty-two female Sprague-Dawley rats were ovariectomized at 8 weeks of age. After 3 months, they were randomly divided into 4 groups and received cement augmentation in the fifth caudal spine with different filler materials; sham-operated rats (S), PMMA (P), CPC (C), and CPC + PRP (CP). Bone mineral density (BMD) and trabecular type-associated morphological parameters, including trabecular bone volume fraction and trabecular thickness in the augmented caudal spine, were evaluated by micro-computed tomography (mirco-CT) 2 weeks after the cementoplasty. Histological analysis was also performed to compare the bone regeneration.

Results: The trabecular bone volume fraction in the CP group was significantly greater than those of all the other groups. Trabecular thickness was higher in the CP group than the $\mathrm{S}$ and $\mathrm{P}$ groups. This augmented trabecular structure in the CP group accordingly showed higher BMD. Histological evaluations showed significantly more bone regeneration in the CP group.

Limitations: There has been a concern that the effect of PRP would be dependent on the species, and might show different results in humans. Baseline values of micro-CT analysis were not measured, which could have provided exact evidence of the changes in trabecular microarchitecture parameters and cement resorption profiles. Finally, caudal vertebrae with filler materials used in biological study should have been compared by their mechanical properties using biomechanical evaluations for a more coherent study, which was not possible due to technical problems.

Conclusions: Incorporating PRP into CPC could accelerate osteoconduction in the augmented vertebra leading to improvement of trabecular bone microarchitecture and BMD in rats.

Key words: Bone mineral density, calcium phosphates, cementoplasty, histology, osteoconduction, osteoporosis, platelet-rich plasma, polymethyl methacrylate, vertebra

Pain Physician 2014; 17:E737-E745 
A $s$ the aging population increases each year, the rising prevalence of osteoporosis-related injuries has become quite an important medical issue. Vertebral body fracture is one of the most common fractures associated with osteoporosis (1). The treatment of osteoporotic vertebral fracture includes bracing and external orthoses, medications, and physical therapy. Another effective treatment option is vertebroplasty, the cement augmentation of fractured osteoporotic bone.

Polymethyl methacrylate (PMMA) bone cement is the most widely used bone substitute for vertebroplasty. The stabilization of an unstable fractured vertebral body by increasing its stiffness and strength and the thermal ablation and/or chemical toxicity of intraosseous neural tissue have been proposed to explain the pain relief after the procedure (2). However, these properties also induce several potential adverse effects, which are adjacent vertebral body fracture from excessive stiffness (3) and thermal and chemical necrosis of surrounding tissues (4).

Calcium phosphate cement (CPC) is highly osteoconductive and is gradually replaced by new bone that can provide substantial improvement in the compressive strength of the osteoporotic or fractured bone (5-7). It reduces the adverse effects of PMMA. The elastic modulus of PMMA is $2700 \mathrm{MPa}$ which is much higher than that of human cancellous bone (168 MPa). In contrast, the elastic modulus of CPC is $180 \mathrm{MPa}$, which is more effective at avoiding the stress shielding effect and abnormal load transfer, as well as reducing secondary fracture of adjacent vertebral bodies (8). PMMA would also induce a significant and dose-dependent reduction of human nucleus pulposus cells, which is significantly more severe than with CPC (4). Intradiscal PMMA leakage is known to be one of the important risk factors in new vertebral body fracture after percutaneous vertebroplasty $(9,10)$. But at the same time, CPC also has some disadvantages. As a crystalline substance, CPC is known to be prone to show failure under shear loading (11). CPC might not provide enough initial stiffness, and therefore progressive and repeated collapse has occurred in the treated vertebra (12). In order to facilitate bone regeneration, growth factors (GFs) are combined with osteoconductive substances.

Platelet-rich plasma (PRP) is considered to be a rich source of GFs, including a high concentration of platelet-derived growth factor (PDGF), transforming growth factor- $\beta 1$ (TGF- $\beta 1$ ), and insulin-like growth factor-1 (IGF-1), which are assigned an important role in bone regeneration (13-18). Since PRP was first introduced in 1998 by Marx et al (13) for maxillofacial reconstructions, extensive attempts were made to promote bone regeneration with PRP, but the results are not consistent (1316). However, though only a few studies investigated the relevance of PRP in osteoporosis, their results revealed that PRP treatment was shown to improve overall bone quality in osteoporotic mice $(17,18)$. According to the other studies which showed that increased bone marrow adipocytes are correlated to decreased bone mineral density in the osteoporotic model $(19,20)$, the underlying mechanism of PRP-mediated treatment is simultaneous up-regulation of osteogenesis and downregulation of adipogenesis.

Caudal vertebrae in rats exhibit bone morphology and remodeling similar to the human adult iliac crest $(21,22)$. Trabecular microfractures were observed in the rat caudal vertebra under cyclic overloading which is known to be correlated to stress or compression vertebral fractures (23). The caudal vertebrae in ovariectomized rats exhibit pathologic bone changes consistent with osteoporosis, and represent a valuable and costeffective model for evaluating the performance of potential bioresorbable bone cements engineered for use with osteoplasty (24). Previous studies demonstrated that a substantial effect of PRP might be observed at 2 weeks $(25,26)$. The total bone area and bone formation rate at 2 weeks were much higher than at 4 weeks for maxillary sinus augmentation with PRP incorporated into bone grafts in a rabbit model (25). Also, in combination with autologous bone applied in the forehead area of pigs, a significant accelerating effect of PRP on early bone regeneration (2 weeks) was shown (26).

The purpose of this study is to compare the early effects of bone filler materials including PMMA, CPC, and $C P C+P R P$ in the ovariectomized rat caudal vertebroplasty model.

\section{Methods}

\section{Preparation}

\section{Animals' Preparation}

At age 8 weeks, 32 female Sprague Dawley rats were ovariectomized and were divided equally into 4 experimental groups (8 in each group), which are Sham (S), PMMA (P), CPC (C), and CPC + PRP (CP) groups. Two additional rats were used as blood donors to obtain PRP. Three months after ovariectomy, they weighed approximately 350 - $400 \mathrm{~g}$. All surgical procedures were performed 
according to the protocol approved by Pusan National University Institutional Animal Care and Use Committee.

\section{PRP Preparation}

Approximately $8 \mathrm{~mL}$ of whole blood was withdrawn using cardiac puncture into an EDTA-HEPES saline containing tube to prevent coagulation in the ovariectomized rats. PRP was prepared after 2 centrifugations (Combi-514R, Hanil, Seoul, Korea) at $200 \mathrm{~g}$ for 10 minutes to separate the plasma portions from the red blood cell fraction, and at $400 \mathrm{~g}$ for 15 minutes to separate the PRP from the platelet-poor plasma. The platelet count was conducted in the whole blood and PRP using a hematology analyzer.

\section{Bone Filler Materials and Surgical Procedure}

Before surgery, anesthesia was induced with $5 \%$ isoflurane in an induction box and maintained with $2.5 \%$ isoflurane via a mask. The animals were infiltrated with $1 \%$ lidocaine to the incision site to reduce the operative pain.

A tourniquet was introduced at the highest point of the tail. Under sterile conditions, a posterior midline incision was made along the proximal tail exposing the dorsal view of the caudal vertebral bodies at the level of the fifth caudal vertebra. After dissecting posterior tendons and dorsal segmental muscles bilaterally, a cavity-like defect (diameter $2 \mathrm{~mm}$, depth $2 \mathrm{~mm}$ ) was made by a round bone bur at the distal $1 / 3$ of the caudal vertebral body. When trabecular bone was exposed, it was gently curetted.

PMMA (Surgical Simplex P, Striker Orthopedics, Mahwah, NJ) and CPC (Polybone, Kyungwon Medical, Seoul, Korea) were prepared according to the manufacturer's instructions. Prior to implantation, in the $C P$ group, the CPC was soaked and kneaded with PRP and solvent (10:1:1 volume ratio) for 5 minutes. Each defect was filled with different bone filler materials and the materials gently compressed with a bone tamp. In the $S$ group, the same defects were created in the same place but were not filled with any bone filler material. The posterior tendons and dorsal segmental muscles were repositioned and closed.

\section{Evaluation of the Results}

To compare the effects of the 3 different bone materials, initially high-resolution micro-computed tomography (micro-CT) imaging was performed for the evaluation of the trabecular bone microarchitectures and bone mineral density (BMD). Secondly, histological analysis was performed to determine bone regeneration in each group.

\section{Micro-CT Scan Analysis}

Two weeks after cement injection, micro-CT scanning was performed on the fifth caudal vertebra of each group. After isoflurane anesthesia, all micro-CT scans were performed with a Siemens Inveon Small Animal micro-PET/CT scanner (Siemens Medical Solutions, Siemens Healthcare Molecular Imaging, Malvern, PA). For each sample, a total of 512 micro-tomographic slices were obtained to scan the whole defect and the surrounding bone. From the micro-CT scan datasets, 3-D models were reconstructed for morphological analysis using the Siemens Inveon Research Workplace Software (IRW, version 3.0, Siemens Medical Solutions, Siemens Healthcare Molecular Imaging, Malvern, PA).

The region of interest ( $\mathrm{ROI}$ ) was selected as the cylinder shape, with a diameter of $1.6 \mathrm{~mm}$ and a height of $2.5 \mathrm{~mm}$, running along the inner edge of the defect or cement as an axis, so that a similar cement volume would be included. The controlling threshold of the trabecular bone and bone cements were separated due to a different threshold. Morphological analysis of the ROI was determined for the vertebral body, including bone volume fraction (BV/TV), trabecular thickness (Tb. Th), trabecular number (Tb.N), and trabecular separation (Tb.Sp) (27). Additionally, the cement volume (CV) fraction in the ROI was calculated. The BMD of the vertebral bodies were also determined after subtraction of the cement data.

\section{Histological Analysis}

After micro-CT imaging, rats were euthanized using carbon dioxide. All fifth caudal vertebrae were harvested and fixed in 10\% formalin. For morphological analysis, they were decalcified in 14\% EDTA for 3 days and subjected to paraffin-embedding. Specimens were then cut and milled into $10-\mu \mathrm{m}$ axial sections and stained with hematoxylin and eosin.

A fixed rectangular $\mathrm{ROI}$, including the surgical area, was chosen in each slide at the same magnification (40 X objective). In the ROI, the degree of osteogenesis was classified into 3 categories: fibrosis and chronic inflammation, mineralization, and new bone formation. All the grading was done in a blind fashion by the pathologist.

\section{Statistical Analysis}

Data were expressed as a median (interquartile range) or a number (\%). For continuous variables, a Kruskal-Wallis test with a post hoc Mann-Whitney $U$ test was performed, while a Chi square test was performed for categorical variables. When multiple 
comparisons for post hoc tests were performed, Bonferroni correction was used to determine the statistical significance between the experimental groups. For Bonferroni correction, a $P$ value was considered to be significantly if less than $0.0083(0.05 / 6)$, otherwise less than 0.05 . All analyses were performed using SPSS statistics 18.0 (SPSS Inc., Chicago, IL).

Table 1. Clinical results during study period.

\begin{tabular}{|c|c|c|c|c|c||}
\hline \multirow{2}{*}{ Group } & \multirow{2}{*}{$\begin{array}{c}\text { Bone } \\
\text { filler } \\
\text { materials }\end{array}$} & \multicolumn{2}{|c|}{ Baseline } & \multicolumn{2}{c|}{$\begin{array}{c}\text { 3 months after } \\
\text { ovariectomy }\end{array}$} \\
\cline { 3 - 6 } & & Number & Weight, g & Number & Weight, g \\
\hline S & None & 8 & $198(195-202)$ & 7 & $342(316-383)$ \\
\hline P & PMMA & 8 & $198(194-204)$ & 7 & $352(346-374)$ \\
\hline C & CPC & 8 & $199(193-210)$ & 7 & $339(328-379)$ \\
\hline CP & CPC+PRP & 8 & $202(194-208)$ & 8 & $343(330-370)$ \\
\hline
\end{tabular}

Data are expressed as median (interquartile range). PMMA, polymethyl methacrylate; CPC, calcium phosphate cement; PRP, platelet-rich plasma; S, sham; P, PMMA; $\mathrm{C}$, calcium phosphate cement; $\mathrm{CP}$, calcium phosphate cement + platelet-rich plasma.

\section{Results}

Thirty-two rats were used in this study, 3 died during and after surgery. The remaining 29 rats had an uneventful recovery and gained weight after the bilateral ovariectomy (Table 1). The platelet count of the first rat was $4.12 \times 10 \% / \mathrm{mL}$ in PRP and $1.08 \times$ $10^{9} / \mathrm{mL}$ in whole blood. The platelet count of the second rat was $4.01 \times 10^{9} / \mathrm{mL}$ in PRP and $1.15 \times 10 \% / \mathrm{mL}$ in whole blood.

\section{Micro-CT Scan Analysis}

Two weeks after surgery, newly formed bone showed in the $\mathrm{C}$ and $\mathrm{CP}$ groups (Fig. 1). CPC had been partly absorbed and replaced by newly formed trabecular bone. In the CP group, newly formed trabecular bone was found not only at the periphery but also in the central area of the cement.

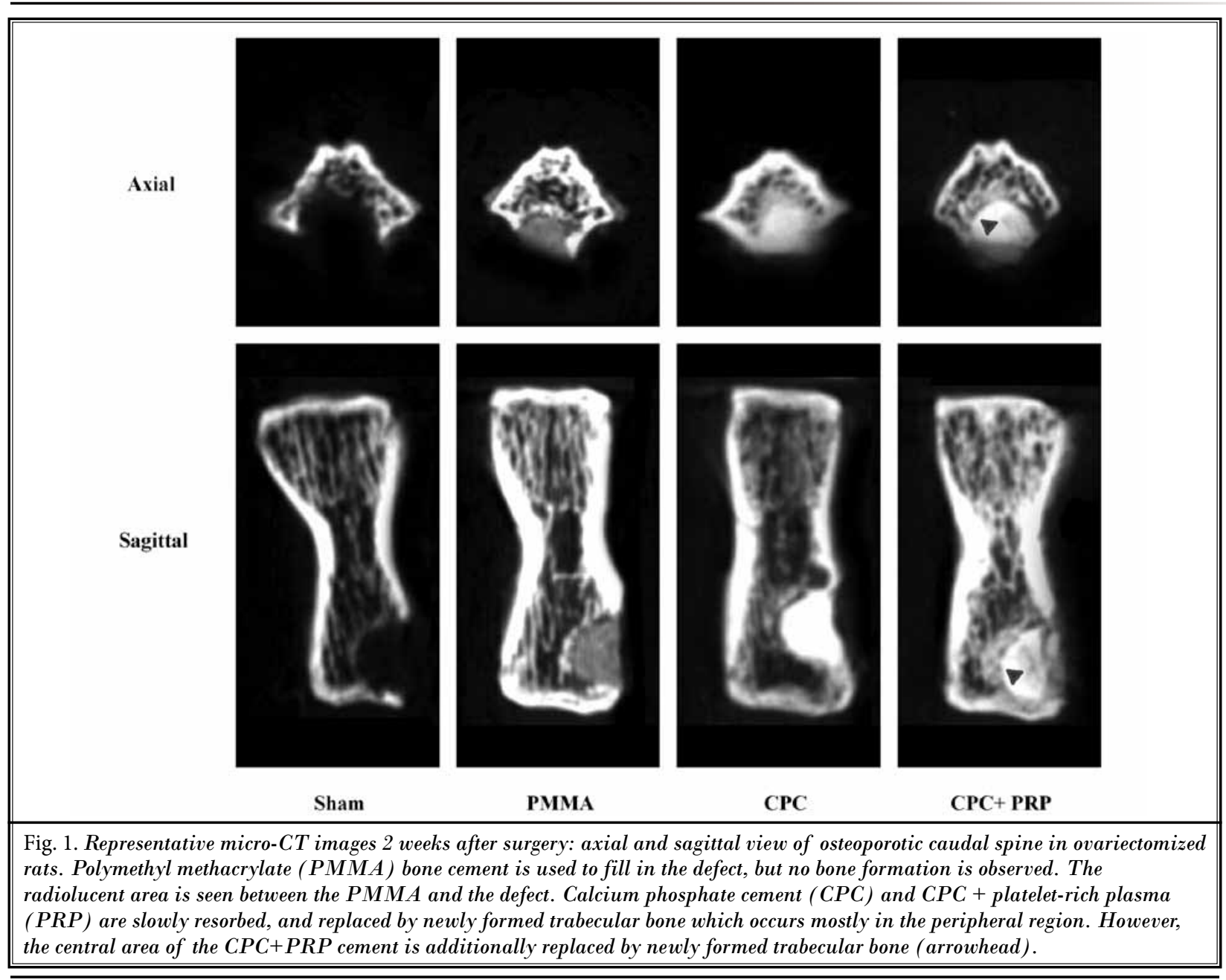


The trabecular bone morphology parameters from micro-CT analysis are shown in Fig. 2. The trabecular
BV/TV of the CP group is significantly greater than that of all the other groups $(P<0.001$ compared to the $S$

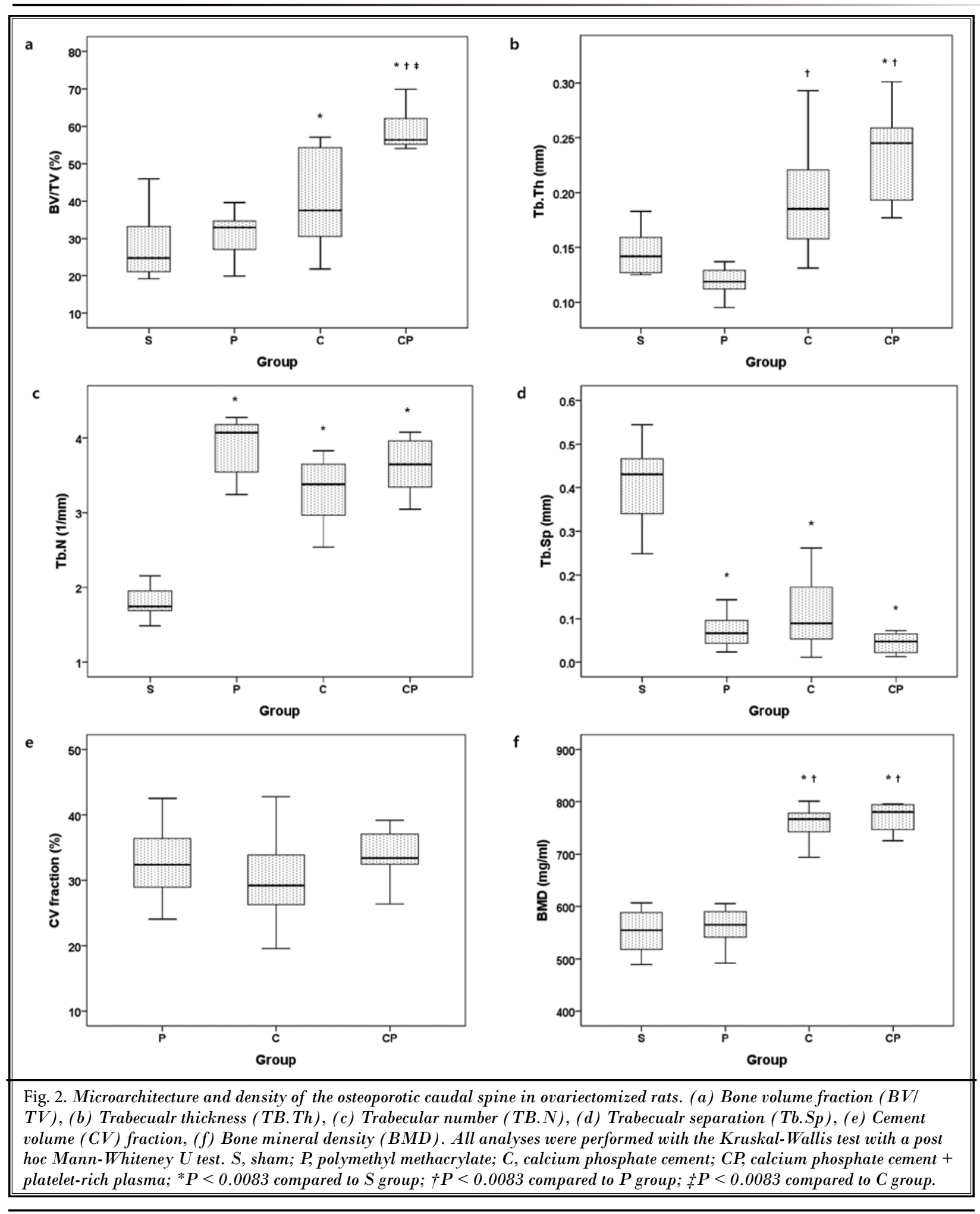


group, 0.001 compared to the $P$ group, 0.006 compared to the $($ group). Tb. Th is higher in the $\mathrm{CP}$ group than the $S$ and $P$ groups $(P=0.002$ compared to $S$ group, $<$ 0.001 compared to $P$ group). This augmented trabecular structure in the $\mathrm{CP}$ group is in accordance with the higher BMD ( $P<0.001$ compared to the $S$ and $P$ groups). However, Tb.N and Tb.Sp were not different in the 3 cement implanted groups, only showing marked difference from the $S$ group $(P=0.002$ compared to the $P$ and C groups, 0.001 compared to the CP group for Tb.N and $P=0.001$ compared to the $P$ group, 0.002 compared to the $C$ groups, $<0.001$ compared to the CP group for $\mathrm{Tb} . \mathrm{Sp}$ ). There is no difference in the $\mathrm{CV}$ fraction among the $P, C$, and $C P$ groups.

\section{Histological Analysis}

Two weeks after implantation, a combination of CPC and PRP yielded the best performance in bone formation in histological examination compared to all the other groups (Fig. 3). All slides of the CP group showed evidence of new bone formation. In the C group, 2 of the 7 slides showed new bone formation and the others only showed mineralization. In contrast, in the $S$ and $P$ groups, there was no obvious indication of new bone formation, and chronic inflammation with fibrous tissue was observed. The data obtained with the histological grading system are shown in Table 2. Statistical analysis indicated that the CP group has a significantly higher grade of osteogenesis than the other groups.

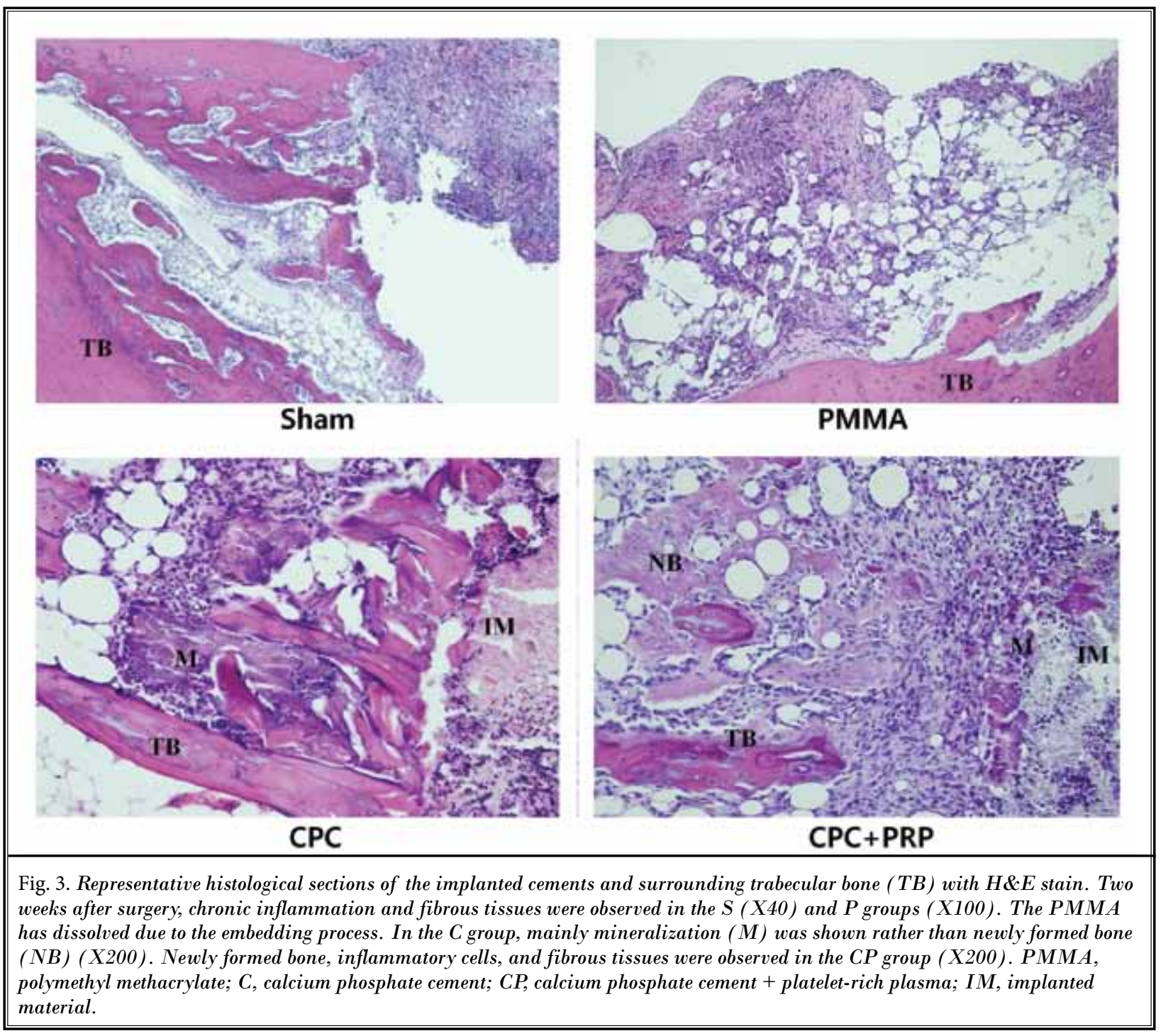




\section{Discussion}

The aim of this study was to investigate whether CPC mixed with PRP can be used as an alternative filler material to PMMA and CPC in vertebroplasty, overcoming their disadvantages, such as adjacent vertebral fracture and early vertebral body recollapse, respectively. The histological and radiological results demonstrated that PRP in combination with CPC accelerates new bone formation in osteoporotic rat models.

Vertebroplasty using CPC has been successfully used as a minimally invasive therapeutic strategy for the treatment of osteoporotic vertebral fracture (5-7). CPC is an osteoconductive biomaterial that is more biocompatible than PMMA. It has been reported that subsequent fractures of the adjacent vertebral body are frequent after vertebroplasty with PMMA, which is assumed to be caused by increased stiffness $(3,28)$. To overcome this potential complication, low modulus, low viscosity, and more biocompatible PMMA have been introduced (29-31). However, more to the mechanical complication, chemical toxicity of PMMA reduces human nucleus pulposus cells which is much severe than CPC (4).

In spite of the advantages, clinical application of CPC is limited by its mechanical weakness $(32,33)$. Incorporating various additives has been a strategy to overcome its poor mechanical performance $(33,34)$. To accelerate replacement of CPC by new bone formation leading to an early acquisition of adequate compressive strength, PRP would be a powerful additive, a rich source of autologous GFs, and would enhance bone regeneration.

Considerable research has demonstrated that PRP accelerates new bone formation $(13,14,17,26)$. In spite of the experimental evidence, its use is still a subject of controversy $(15,16,25)$. Several explanations for this debate have been suggested. First of all, the interval between implantation and investigation is too long to prove the impact of PRP on bone regeneration. Because the life span of PRP and growth factors is short, it is better to observe the effect of PRP on bone regeneration during early stages of bone healing $(35,36)$. Secondly, the PRP concentration needs to be measured. A medium-concentration of PRP (2.5 - $4.5 \times 109 / \mathrm{mL})$ promotes bone regeneration. Higher concentrations of PRP would inhibit osteogenic proliferation, and a lower concentration of PRP would have no effect on osteogenesis $(37,38)$. In the present study, a medium concentration of PRP was used with CPC and the effects were investigated at 2 weeks after operation.
Table 2. Degree of osteogenesis.

\begin{tabular}{||l|l|l|l|}
\hline \multirow{2}{*}{ Group } & \multicolumn{3}{|c|}{ Degree of Osteogenesis } \\
\cline { 2 - 4 } & $\begin{array}{c}\text { Fibrosis } \\
\text { and chronic } \\
\text { inflammation }\end{array}$ & Mineralization & $\begin{array}{l}\text { New bone } \\
\text { formation }\end{array}$ \\
\hline $\mathrm{S}$ & $7(100)$ & $0(0)$ & $0(0)$ \\
\hline $\mathrm{P}$ & $7(100)$ & $0(0)$ & $0(0)$ \\
\hline $\mathrm{C}^{*} \dagger$ & $0(0)$ & $5(71.4)$ & $2(28.6)$ \\
\hline $\mathrm{CP}^{*} \dagger \ddagger$ & $0(0)$ & $0(0)$ & $8(100)$ \\
\hline
\end{tabular}

Data was expressed as number (\%). S, sham; P, polymethyl methacrylate; $\mathrm{C}$, calcium phosphate cement; $\mathrm{CP}$, calcium phosphate cement + plateletrich plasma. ${ }^{\star} P<0.0083$ compared to $S$ group; $\uparrow P<0.0083$ compared to $P$ group; $\ddagger P<0.0083$ compared to $C$ group.

There was significantly higher new bone formation in the CP group compared with all the other groups, in both micro-CT and histological evaluation. The morphological results from micro-CT analysis represented an overall increase of trabecular structure in the CP groups. Interestingly, Tb.N and Tb.Sp were not different in the 3 cement implanted groups, despite a higher BV/TV and Tb.Th in the CP group. This can be explained by a recent report that a mechanism of bone apposition is to build upon the existing microarchitecture rather than by increasing the number of trabeculae (39). Based on this study, new trabecular bone formation could occur by closing inter-trabecular spaces which leads to a lower Tb.Sp. Nevertheless, the muted changes in Tb.Sp along with an increase in Tb.Th can be assumed to show that it was too early for significant change in Tb.Sp in the present study.

There have been a few investigations about the effects of CPC and PRP mixture on bone regeneration. $A$ recent report (40) demonstrated that the bone tissue regeneration of the CPC with PRP group showed a significantly larger area of new bone formation compared with the CPC only group at 9 weeks post-operation in rabbits. However, in terms of the early effect of PRP, there is a report (16) that PRP had no effect on the early stages of cranial defect healing in rats. There are a number of possible explanations for this discrepancy in the effect of PRP-added CPC. First, the defect size was different. Smaller injuries heal faster. Plachokova et al (16) made a $6.2 \mathrm{~mm}$ defect, while a $2.0 \mathrm{~mm}$ defect was made in our study. Second, normal rats were used in their study, however; osteoporotic rats were used in the present study. Recently, effects of PRP on osteoporosis have been investigated $(17,37)$. In those studies, PRP enhanced osteogenesis in ovariectomized mice and even treated osteoporosis. Also, PRP promoted osteoporotic 
fracture repair in the long bones of ovariectomized rats at 2,4 , and 8 weeks postoperatively.

According to this study, the incorporation of PRP into CPC can accelerate trabecular bone formation in osteoporosis. The improvement of trabecular microarchitecture parameters can lead to an increase in bone strength. There have been several studies showing that trabecular microarchitecture and bone strength are significantly correlated $(41,42)$. In these studies, ultimate stress, which is the most important mechanical property related to clinical fracture, was strongly correlated with BV/TV and Tb.Th. Although PRP + CPC itself has been known to have lower modulus compared with CPC (40), its ability of releasing GFs accelerates bone regeneration which can lead to improved bone quality and strength.

There are some limitations that should be considered in this study. This animal study used rats. There has been a concern that the effect of PRP would be dependent on the species (43). And no standardized methods to prepare rat PRP, such as critically effective amounts of platelets or levels of GFs, have been published. Baseline values of micro-CT analysis were not measured, which could have provided exact evidence of the changes in trabecular micro-architecture parameters and cement resorption profiles. Finally, caudal vertebrae with filler materials used in biological study should have been compared by their mechanical properties using biomechanical evaluations for a more coherent study, which was not possible due to technical problems.

\section{Conclusion}

In conclusion, the incorporation of PRP into CPC could accelerate osteoconduction in the augmented vertebra leading to improvement of trabecular bone microarchitecture and BMD in rats. Further studies are needed to prove the results that CPC with PRP would be a good alternative to PMMA and CPC, including biomechanical and clinical investigations.

\section{References}

1. Cooper C, Melton LJ 3rd. Vertebral fractures. BM] 1992; 304:1634-1635.

2. Lieberman IH, Togawa D, Kayanja MM. Vertebroplasty and kyphoplasty: Filler materials. Spine ] 2005; 5:305S-316S.

3. Chang CY, Teng MM, Wei CJ, Luo CB, Chang FC. Percutaneous vertebroplasty for patients with osteoporosis: A one-year follow-up. Acta Radiol 2006; 47:568-573.

4. Lazary A, Speer G, Varga PP, Balla B, Bacsi K, Kosa JP, Nagy Z, Takacs I, Lakatos $P$. Effect of vertebroplasty filler materials on viability and gene expression of human nucleus pulposus cells. J Orthop Res 2008; 26:601-607.

5. Bai B, Jazrawi LM, Kummer FJ, Spivak JM. The use of an injectable, biodegradable calcium phosphate bone substitute for the prophylactic augmentation of osteoporotic vertebrae and the management of vertebral compression fractures. Spine (Phila Pa 1976) 1999; 24:1521-1526.

6. Belkoff SM, Mathis JM, Erbe EM, Fenton DC. Biomechanical evaluation of a new bone cement for use in vertebroplasty. Spine (Phila Pa 1976) 2000; 25:1061-1064.

7. Heini PF, Berlemann U, Kaufmann M, Lippuner K, Fankhauser C, van Landuyt P. Augmentation of mechanical proper- ties in osteoporotic vertebral - bones - a biomechanical investigation of vertebroplasty efficacy with different bone cements. Eur Spine J 2001; 10:164-171.

8. Yang H, Zou J. Filling materials used in kyphoplasty and vertebroplasty for vertebral compression fracture: A literature review. Artif Cells Blood Substit Immobil Biotechnol 2011; 39:87-91.

9. Lin EP, Ekholm S, Hiwatashi A, Westesson PL. Vertebroplasty: Cement leakage into the disc increases the risk of new fracture of adjacent vertebral body. AJNR Am J Neuroradiol 2004; 25:175-180.

10. Komemushi A, Tanigawa N, Kariya S, Kojima H, Shomura Y, Komemushi S, Sawada S. Percutaneous vertebroplasty for osteoporotic compression fracture: Multivariate study of predictors of new vertebral body fracture. Cardiovasc Intervent Radiol 2006; 29:580-585.

11. Wilke HJ, Mehnert U, Claes LE, Bierschneider MM, Jaksche $H$, Boszczyk BM. Biomechanical evaluation of vertebroplasty and kyphoplasty with polymethyl methacrylate or calcium phosphate cement under cyclic loading. Spine (Phila Pa 1976) 2006; 31:2934-2941.

12. Heo DH, Kuh SU. Progressive, repeated lumbar compression fracture at the same level after vertebral kyphoplasty with calcium phosphate cement. Case report. J
Neurosurg Spine 2007; 6:559-562.

13. Marx RE, Carlson ER, Eichstaedt RM, Schimmele SR, Strauss JE, Georgeff KR. Platelet-rich plasma: Growth factor enhancement for bone grafts. Oral Surg Oral Med Oral Pathol Oral Radiol Endod 1998; 85:638-646.

14. Marx RE. Platelet-rich plasma: Evidence to support its use. J Oral Maxillofac Surg 2004; 62:489-496.

15. Jungbluth $P$, Wild $M$, Grassmann JP, Ar $E$, Sager $M$, Herten $M$, Jager $M$, Becker J, Windolf J, Hakimi M. Platelet-rich plasma on calcium phosphate granules promotes metaphyseal bone healing in mini-pigs. J Orthop Res 2010; 28:1448-1455.

16. Plachokova AS, van den Dolder J, Stoelinga PJ, Jansen JA. Early effect of platelet-rich plasma on bone healing in combination with an osteoconductive material in rat cranial defects. Clin Oral Implants Res 2007; 18:244-251.

17. Liu HY, Wu AT, Tsai CY, Chou KR, Zeng R, Wang MF, Chang WC, Hwang SM, Su CH, Deng WP. The balance between adipogenesis and osteogenesis in bone regeneration by platelet-rich plasma for age-related osteoporosis. Biomaterials 2011; 32:6773-6780.

18. Lo WC, Chiou JF, Gelovani JG, Cheong $\mathrm{ML}$, Lee $\mathrm{CM}$, Liu HY, Wu CH, Wang 
MF, Lin CT, Deng WP. Transplantation of embryonic fibroblasts treated with platelet-rich plasma induces osteogenesis in SAMP8 mice monitored by molecular imaging. J Nucl Med 2009; 50:765-773.

19. Benayahu D, Shur I, Ben-Eliyahu S. Hormonal changes affect the bone and bone marrow cells in a rat model. J Cell Biochem 2000; 79:407-415.

20. Duque G. Bone and fat connection in aging bone. Curr Opin Rheumatol 2008; 20:429-434.

21. Baron R, Tross R, Vignery A. Evidence of sequential remodeling in rat trabecular bone: Morphology, dynamic histomorphometry, and changes during skeletal maturation. Anat Rec 1984; 208:137-145.

22. Miyakoshi N, Sato K, Abe T, Tsuchida T, Tamura Y, Kudo T. Histomorphometric evaluation of the effects of ovariectomy on bone turnover in rat caudal vertebrae. Calcif Tissue Int 1999; 64:318-324.

23. Kummari SR, Davis AJ, Vega LA, Ahn N, Cassinelli EH, Hernandez CJ. Trabecular microfracture precedes cortical shell failure in the rat caudal vertebra under cyclic overloading. Calcif Tissue Int 2009; 85:127-133.

24. Wang ML, Massie J, Perry A, Garfin SR, Kim CW. A rat osteoporotic spine model for the evaluation of bioresorbable bone cements. Spine J 2007; 7:466-474.

25. Butterfield KJ, Bennett J, Gronowicz G, Adams D. Effect of platelet-rich plasma with autogenous bone graft for maxillary sinus augmentation in a rabbit model. J Oral Maxillofac Surg 2005; 63:370-376.

26. Schlegel KA, Donath K, Rupprecht $S$, Falk S, Zimmermann R, Felszeghy E, Wiltfang J. De novo bone formation using bovine collagen and platelet-rich plasma. Biomaterials 2004; 25:5387-5393.
27. Parkinson IH, Forbes $D$, Sutton-Smith P, Fazzalari NL. Model-independent $3 \mathrm{D}$ descriptors of vertebral cancellous bone architecture. J Osteoporos 2010; 2010:641578.

28. Nouda S, Tomita S, Kin A, Kawahara K, Kinoshita M. Adjacent vertebral body fracture following vertebroplasty with polymethylmethacrylate or calcium phosphate cement: Biomechanical evaluation of the cadaveric spine. Spine (Phila Pa 1976) 2009; 34:2613-2618.

29. Seo SS, Park JY, Kim HJ, Yoon JW, Park $\mathrm{SH}$, Kim KH. Percutaneous osteoplasty for the treatment of a painful osteochondral lesion of the talus: A case report and literature review. Pain Physician 2012; 15:E743-E748.

30. Ri HS, Lee DH, Kim KH. Searching for hidden, painful osteochondral lesions of the ankle in patients with chronic lower limb -pain - two case reports. Korean J Pain 2013; 26:164-168.

31. Boger A, Heini P, Windolf $M$, Schneider E. Adjacent vertebral failure after vertebroplasty: A biomechanical study of low-modulus PMMA cement. Eur Spine J 2007; 16:2118-2125.

32. Lim TH, Brebach GT, Renner SM, Kim WJ, Kim JG, Lee RE, Andersson GB, An HS. Biomechanical evaluation of an injectable calcium phosphate cement for vertebroplasty. Spine (Phila Pa 1976) 2002; 27:1297-1302.

33. Ambard AJ, Mueninghoff L. Calcium phosphate cement: Review of mechanical and biological properties. J Prosthodont 2006; 15:321-328.

34. Perez RA, Kim HW, Ginebra MP. Polymeric additives to enhance the functional properties of calcium phosphate cements. J Tissue Eng 2012; 3:2041731412439555.
35. Schmitz JP, Hollinger JO. The biology of platelet-rich plasma. J Oral Maxillofac Surg 2001; 59:1119-1121.

36. Mundy GR. Regulation of bone formation by bone morphogenetic proteins and other growth factors. Clin Orthop Relat Res 1996; 324:24-28.

37. Chen L, Yang $X$, Huang G, Song D, Ye $\mathrm{XS}, \mathrm{Xu} \mathrm{H}, \mathrm{Li}$ W. Platelet-rich plasma promotes healing of osteoporotic fractures. Orthopedics 2013; 36:e687-e694.

38. Kawasumi M, Kitoh $H$, Siwicka KA, Ishiguro N. The effect of the platelet concentration in platelet-rich plasma gel on the regeneration of bone. J Bone Joint Surg Br 2008; 90:966-972.

39. Campbell GM, Ominsky MS, Boyd SK. Bone quality is partially recovered after the discontinuation of RANKL administration in rats by increased bone mass on existing trabeculae: $A n$ in vivo micro-CT study. Osteoporos Int 2011; 22:931-942.

40. Chen JC, Ko CL, Shih CJ, Tien YC, Chen WC. Calcium phosphate bone cement with $10 \mathrm{wt} \%$ platelet-rich plasma in vitro and in vivo. J Dent 2012; 40:114-122.

41. Teo JC, Si-Hoe KM, Keh JE, Teoh SH. Relationship between CT intensity, micro-architecture and mechanical properties of porcine vertebral cancellous bone. Clin Biomech (Bristol, Avon) 2006; 21:235-244.

42. Mittra E, Rubin C, Qin YX. Interrelationship of trabecular mechanical and microstructural properties in sheep trabecular bone. J Biomech 2005; 38:1229-1237.

43. Plachokova AS, van den Dolder J, van den Beucken JJ, Jansen JA. Bone regenerative properties of rat, goat and human platelet-rich plasma. Int J Oral Maxillofac Surg 2009; 38:861-869. 
